\title{
A perspective on the IEEE 802.11e Protocol for the Factory Floor
}

\author{
Lucia Lo Bello, Emanuele Toscano \\ and Salvatore Vittorio \\ University of Catania \\ Italy
}

\section{Introduction}

In recent years, wireless technologies have been identified as a very attractive option for industrial and factory automation. Some of the benefits of these technologies are mobility support, reduced cabling and installation costs, reduced danger of breaking cables and less hassle with connectors. Typical application classes where wireless technologies might be used are closed-loop control involving mobile subsystems, coordination among mobile robots or autonomous vehicles, health monitoring of machines, tracking of parts and many more. Moreover, wireless technologies may be useful in factory automation systems where there is the need to dynamically connect new components to an already deployed wired communication system that cannot be reached (easily and/or reliably) with a cable.

On the other hand, wireless solutions and products available today on the market are considered unsuitable for implementing distributed control applications and systems, in particular when real-time and/or reliability constraints are key issues. In fact, wireless networks are influenced by a number of factors that have to be carefully analysed before using them at the device level of factory automation systems. As an example, wireless transmissions are quite sensitive to electro-magnetic noise, obstacles, multi-path fading, etc. Industrial environments are generally very hostile and multiple types of noise may cause transmission errors, that can significantly affect the robustness. Moreover, as the wireless channel is non-deterministic and time-varying, the classical deterministic performance measures such as the worst-case transmission times should be replaced by probabilistic assessments.

IEEE 802.11 is the most widely used wireless technology and several research works exist that study its suitability for the industrial environment and try to enhance the support provided to industrial traffic. Moreover, the interest of the industrial community regarding this protocol has noticeably grown since the IEEE 802.11e amendment has been incorporated into the published IEEE 802.11-2007 standard. In fact, such an amendment uses the same IEEE 802.11 physical layer, but provides two novel channel access mechanisms that support Quality of Service (QoS), namely, EDCA and HCCA. This Chapter aims at giving a summary of the many works in literature, to provide a broad overview on the current results regarding the IEEE 802.11 e protocol in industrial communication, with a special 
focus on the support provided to real-time traffic in factory automation. While Section 2 presents a summary of the IEEE 802.11e protocol, Sections 3 and 4 give an overview of analytic performance models and of the most relevant admission control algorithms currently in literature, respectively. In Section 5 some improved scheduling algorithms for HCCA are presented. In addition, as analytic models prove that the EDCA performance strongly depends on the protocol parameters, some mechanisms that control those parameters to improve the performance of the EDCA traffic will be discussed as well. Section 6 discusses several case studies of using 802.11e for factory automation.

\section{The IEEE 802.11 standard}

The 802.11 standard defines the Media Access Control (MAC) and Physical (PHY) layers specifications for wireless LANs. Three different physical layer specifications were originally defined, namely, Frequency Hopping Spread Spectrum (FHSS), Direct Sequence Spread Spectrum (DSSS) and Infrared (IR), with a nominal data rate up to $2 \mathrm{Mbps}$. Today such PHY specifications are obsolete, while three different specifications are currently in use, defined in 802.11a (IEEE 802.11a, 1999), 802.11b (IEEE 802.11b, 1999) and 802.11g (IEEE $802.11 \mathrm{~g}$, 2003). While 802.11a operates in the $5 \mathrm{GHz}$ band supporting a data rate up to 54 Mbps, both the $802.11 \mathrm{~b}$ and $802.11 \mathrm{~g}$ physical layers operate in the license-free $2.4 \mathrm{GHz}$ ISM (Industrial, Scientific and Medical) band, supporting data rates up to $11 \mathrm{Mbps}$ and $54 \mathrm{Mbps}$, respectively.

The IEEE 802.11 protocol defines two different WLAN architectures, i.e., Basic Service Set (BSS) and Independent Basic Service Set (IBSS). In a Basic Service Set, some wireless stations (STAs) are associated to an Access Point (AP) and all the communications take place through the AP. Conversely, in an Independent Basic Service Set, STAs can communicate directly to each other, provided that they are within each other's transmission range. This kind of architecture allows the formation of a wireless ad-hoc network in the absence of any network infrastructure.

At the MAC layer, the IEEE 802.11 protocol defines two different access mechanisms, the Distributed Coordination Function (DCF) and the Point Coordination Function (PCF). The former access mechanism is mandatory and provides distributed channel access based on a Carrier Sense Multiple Access with Collision Avoidance (CSMA/CA). A node willing to transmit a data frame performs the carrier sensing to determine whether the channel is idle or busy. After sensing an idle channel for a duration equal to a Distributed Interframe Space (DIFS) time, the node can transmit. If a collision occurs, the node starts a back-off procedure to avoid other collisions. The back-off procedure starts a random timer, whose value is uniformly chosen in the $[0, \mathrm{CW}]$ interval, that is decremented at each slot time after the channel is sensed idle for a DIFS time. If the medium becomes busy during this back-off process, the station pauses its back-off timer until the channel is free again. The next transmission attempt will occur when the back-off timer expires. In particular, at the first transmission attempt, the $\mathrm{CW}$ is set to the minimum Contention Window size, CWmin. After each unsuccessful transmission, the $\mathrm{CW}$ is increased, using the equation

$$
\mathrm{CW}_{\text {new }}=2 \times\left(\mathrm{CW}_{\text {old }}+1\right)-1 \text {, }
$$


until it reaches the maximum Contention Window size, CWmax. Conversely, after each successful transmission the $\mathrm{CW}$ value is reset to CWmin. The PCF mechanism is optional and divides time in superframes, providing centralized channel access through polling within a contention-free period (CFP) of the superframe. While DCF is designed to support only best effort traffic, PCF may be used for time bounded services. However, in PCF the AP has to contend for channel access at the beginning of each CFP. This leads to unpredictable jitters in the start time of the CFP, that make it difficult to support periodic time-constrained traffic that is typical of industrial applications. Moreover, in PCF the duration of transmissions by the polled station is unknown. To provide a better support for time constrained applications some extensions of the basic MAC protocol are provided by the IEEE 802.11e specifications.

\subsection{The IEEE 802.11e amendment}

The 802.11e standard specification (IEEE 802.11, 2007) was published by the IEEE Task Group E to provide mechanisms to enhance the current 802.11 MAC, so as to provide the support of Quality of Service requirements. In IEEE 802.11e, the DCF remains the principal access method when any QoS support is not needed while, as in IEEE 802.11, the PCF is optional. However, an additional coordination function is introduced, called a Hybrid Coordination Function (HCF), which in turn introduces two additional MAC modes, i.e., Enhanced Distributed Channel Access (EDCA), and HCF Controlled Channel Access (HCCA), the former being an enhancement of Distributed Coordination Function (DCF), while the latter being an enhancement of the Point Coordination Function (PCF).

The IEEE 802.11e protocol defines a Contention Period (CP) and Contention Free Period (CFP), during which the EDCA and the HCCA operate, respectively. These two periods appear alternately, and their durations are configurable according to the application requirements. While the presence of the CFP is optional, as it is only needed when HCCA is used, a minimum CP is always needed, as EDCA is also used to request resources for HCCA traffic. A CFP is delimited by a Beacon frame at the start and by a CF-End frame at the end. The frame exchange mechanism of IEEE 802.11e is similar to that of IEEE 802.11, but some novel features have been introduced, e.g., the possibility to attach either an ACK or a poll for a specific QoS Station (QSTA) to a previously received frame (piggybacking). In this way the number of frames to be exchanged is reduced and the overhead is decreased. In IEEE 802.11e, the time interval during which a particular non-AP station has the right to access the channel is called transmission opportunity (TXOP). A TXOP is characterized by a starting time and a maximum duration. Two different types of TXOPs exist: EDCA TXOP and HCCA TXOP. The former is obtained by means of the EDCA during the CP, while the latter is assigned by the Hybrid Coordinator $(\mathrm{HC})$ during either the CFP or a special part of the $\mathrm{CP}$, called Controlled Access Phase (CAP).

A minimum interval between the transmission of consecutive frames is defined, namely the Inter Frame Space (IFS). While different IFS types exist in the IEEE 802.11 protocol to prioritize different kinds of frames, in IEEE 802.11e IFSs are also used to prioritize channel access of data frames belonging to different service categories, by introducing an Arbitration IFS (AIFS). 


\subsection{HCF Controlled Channel Access (HCCA)}

Similarly to PCF, HCCA provides polled access to the wireless medium. But unlike PCF, QoS polling can take place during $\mathrm{CP}$ and scheduling of packets is based on the traffic requirements. In particular, when a station (QSTA) wants to associate with a certain Basic Service Set (BSS), it specifies its requirements during the so-called TSPEC negotiation. The QoS stations (QSTAs) send QoS reservation requests using a special QoS management frame, called Traffic Specification (TSPEC). The TSPEC frame contains the set of parameters that define the QoS characteristics (such as mean data rate or delay bound) of the particular traffic they are willing to transmit. An Admission Control Unit (ACU) in the QoS Access Point (QAP) is in charge of admitting or rejecting a new stream based on both the resource available and scheduling values.

The time between two consecutive beacon frames is called a super-frame. It is divided into a contention free period (CFP) and a contention period (CP) as shown in Fig. 1.

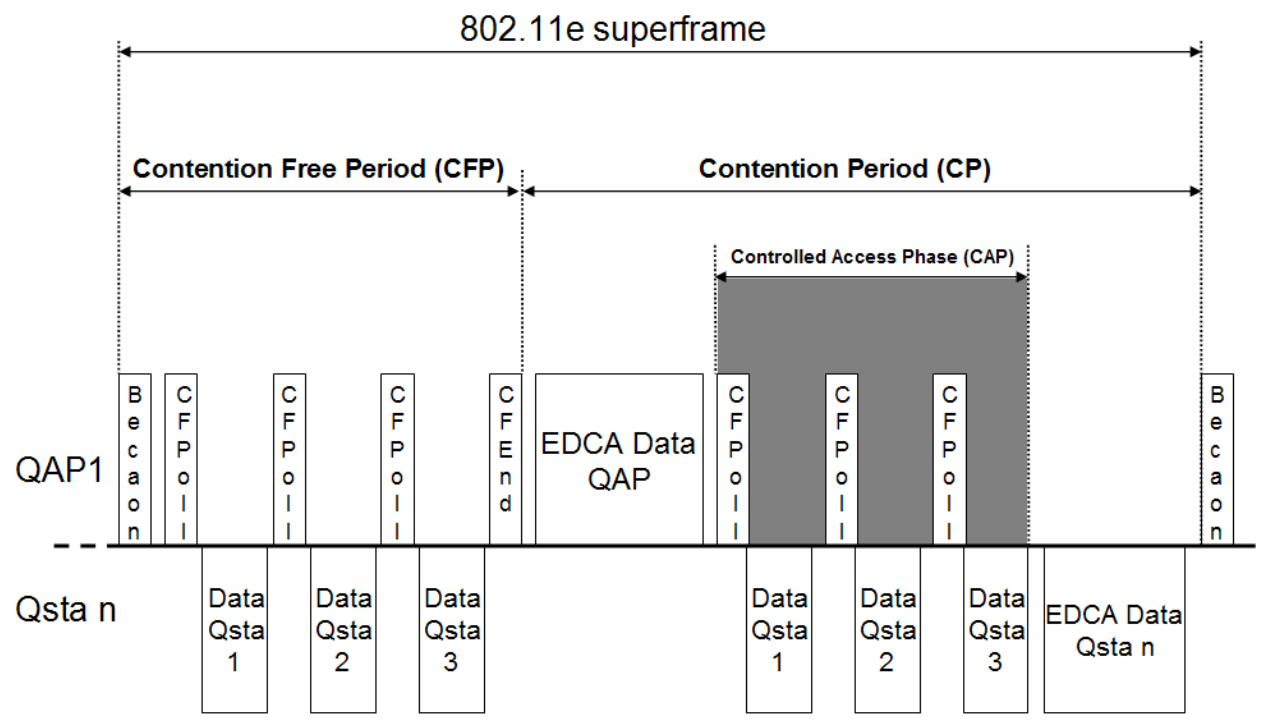

Fig. 1. 802.11e Super-frame

During CFP, the hybrid coordinator (HC) controls the access to the channel by polling its associated stations through CF-Poll messages, according to their QoS requirements. The HC will typically reside within an 802.11e AP.

HCCA can be used not only in the CPF but also in CP together with EDCA, i.e., the HC can poll for data even during the $\mathrm{CP}$. The part of the $\mathrm{CP}$ accessed using polling is called Controlled Access Phase (CAP). The presence of a CAP is possible thanks to the fact that, before accessing the channel, each station has to wait for an interframe space. As the interframe space of the $\mathrm{HC}$ is shorter than those of other stations, the HC has priority over any QSTA and it can allocate TXOP either to itself whenever it has a frame to send or to some other stations allowing them to deliver as many data frames as can be accommodated into the time allocated by the TXOP. As the HC (Hybrid Coordinator) allocates the TXOPs 
through a polling, a scheduler is needed to select the order in which stations are to be polled. The IEEE 802.11e standard does not impose a mandatory HCCA scheduling algorithm, but it offers a reference scheduler that respects a minimum set of performance requirements, on the basis of the mean data rate, nominal MAC Service Data Unit (MSDU) size and either maximum service interval or delay bound information provided by the TSPEC.

\subsection{Enhanced Distributed Channel Access (EDCA)}

The EDCA mode extends the IEEE 802.11 Distributed Coordination Function (DCF) (IEEE 802.11, 2007) by differentiating traffic into four Access Categories (ACs): AC_BK (background category), AC_BE (best-effort category), AC_VI (video category) and AC_VO (voice category), where AC_VO is the highest priority category, while AC_BK is the lowest. To manage the different ACs, EDCA implements in each node a dedicated transmit queue and an independent back-off entity for each AC. Each queue works as an independent DCF station and uses its own parameter set, including the Arbitration Inter-Frame Space (AIFS), the minimum Contention Window size (CWmin), the maximum Contention Window size (CWmax) and the Transmission Opportunity limit (TXOPlimit), that is the time duration a station may transmit after winning access to the medium. Similarly to an 802.11 DCF node, each AC starts a back-off timer after sensing an idle channel for a duration equal to an AIFS length. However, while in DCF all nodes have the same probability to access the channel, in EDCA the AIFS depends on the AC, so that its duration is shorter for the higher priority ACs, which thus have a higher probability of accessing the channel than the lower ACs (Fig. 2).

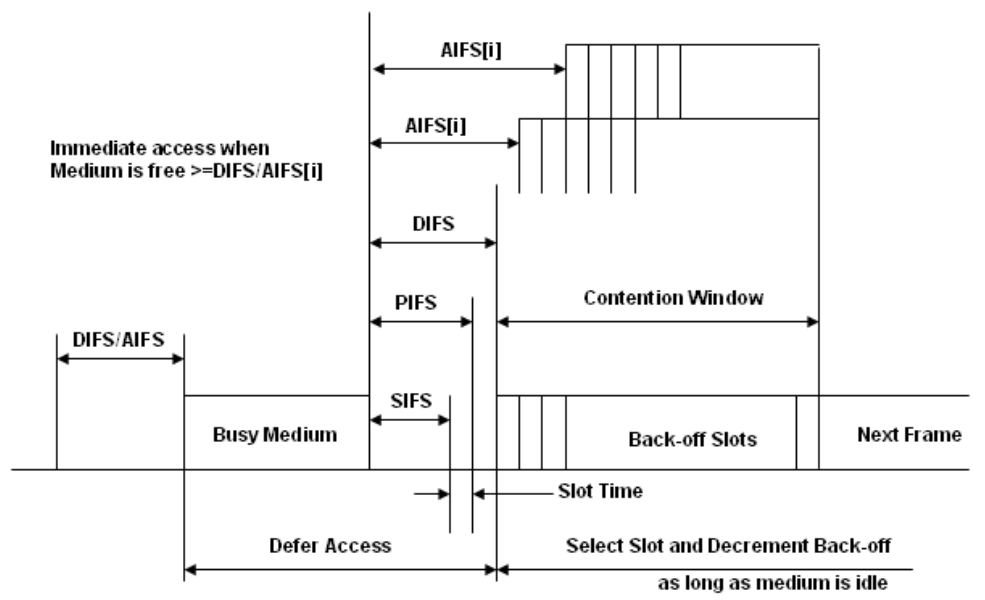

Fig.2. Inter Frame spaces in the EDCA mechanism, redrawn from (IEEE 802.11, 2007).

\subsection{EDCA vs. HCCA in industrial environments}

The nature of the two MAC protocols (EDCA and HCCA) is very different. 
The HCCA protocol is the most suitable for real-time traffic, as the polling-based scheme can support time constrained applications, even though only statistic guarantees can be provided, due to the non-deterministic nature of the channel. HCCA is a centralized protocol, so the Access Point can adopt suitable scheduling and admission control algorithms to allocate at the MAC layer the timeslots needed to meet the station requirements.

On the other hand, EDCA is a protocol which provides differentiated services to different access categories, in which it is not possible to allocate bandwidth to the applications. Therefore, EDCA cannot be adopted in safety-critical systems, and not even for applications with tight timing constraints. But, in many practical situations in industrial systems, the occurrence of a missing frame does not affect human safety or the safety of production equipment. In such cases it is sufficient to ensure that the transmission deadlines are met most of the times. Hence, a number of works exist in literature that study the performance of the EDCA protocol in different situations (e.g. saturation and non-saturation). Such studies can be useful in implementing some admission control algorithms that allow to meet the time requirements in a probabilistic way.

\section{Analytic models of IEEE 802.11e}

\subsection{Analytic models of EDCA}

Several theoretical EDCA performance analyses exist in literature. All of them are implicitly or explicitly derived from analytic models of the 802.11 DCF.

As the theoretical analysis can potentially be complex, a proper set of assumptions is needed to obtain a simple yet accurate analytic model. Most of the models assume that every station has always backlogged data ready to be transmitted in its buffer at any time (in saturation). Other models release the saturation assumption. As a result, we categorized these works in two groups, i.e., models "under saturation" and "under non-saturation" conditions.

\subsubsection{Analytic model under saturation condition.}

Three major analytic models have been proposed to analyse the performance of CSMA/CA DCF scheme in saturation.

1. Bianchi (Bianchi, 2000) proposed an analytical evaluation, where the behaviour of a single station is modelled with a simple Discrete Time Markov Chain (DTMC). In this analysis, the author makes the assumption of constant and independent collision probability of a packet transmitted by each station, regardless of the number of retransmissions already suffered. The outcome of the Markov model is the stationary probability that a station will transmit in a generic slot time. Then, the saturation throughput is expressed as a function of such a stationary probability, by studying the events that may occur in a generic slot time.

2. Calì et al. (Calì et al., 1998 \& 2000) use the renewal theory to analyse a p-persistent variant of DCF, which differs from the standard protocol only in the selection of the back-off interval. In particular, the p-persistent IEEE 802.11 protocol samples its back-off interval from a geometric distribution with parameter $p$, while in the standard a binary exponential back-off is used. This model is able to quantify the maximum achievable throughput as a function of the protocol parameters, e.g., the contention window size. Moreover, the authors showed by means of simulations 
that the p-persistent protocol closely approximates IEEE 802.11 when the average back-off interval is the same.

3. Tay and Chua (Tay \& Chua, 2001) make some simplifications in the 802.11 MAC and model it with an average value mathematical model to evaluate DCF back-off procedure and to calculate the average number of interruptions that the back-off timer experiences. This model assumes slot homogeneity, i.e., constant collision probability at an arbitrary back-off slot, to provide closed-form expressions for the collision probability and the saturation throughput, thus facilitating the analysis of the dependence of system performance on the protocol parameters. The model validation showed that, even though this model omits many system details, it still achieves good accuracy.

These models are used and extended (especially Bianchi's work) to model the EDCA scheme and to include its extra features.

Xiao (Xiao, 2004 \& 2005) modified the Markov chain in (Bianchi, 2000) to model resource sharing by different access categories and to analyse the CW size differentiation in the EDCA mechanism. This model is able to derive saturation throughput, delays and frame dropping probabilities of the different priority classes. It differentiates the minimum backoff window size, the back-off window-increasing factor and the retransmission limit of the different access categories. However, IFS-based priority is not taken into account.

The work in (Robinson \& Randhawa, a \& b 2004) keeps the two dimensional Markov chain, but takes into account that different contentions can experience different collision probabilities because the probability that a transmission collides is a function of the size and composition of the set of competing stations. Moreover, they perform an analysis on backoff and transmission after a collision occurs.

In (Kong et al., 2004), the authors took into account AIFS differentiation via a 3-dimensional DTMC. They introduced a separate one-dimensional Markov chain to be used along with Bianchi's model, so that the model can reflect the back-off and access procedures accurately. Moreover this model takes into account the back-off timer freeze that occurs when a station is deferring, and the virtual collision policy. They analyze the throughput performance of differentiated service traffic and propose a recursive method capable of calculating the mean average delay.

In (Inan et al., 2008), the authors analysed the saturation throughput of EDCA by means of a DTMC which models AIFS and CW differentiation between the ACs in the constant transmission probability assumption. Moreover they showed that the slot homogeneity assumption does not lead to accurate performance prediction when the saturation assumption is released.

Another analytical model based on a three-dimensional DTMC is proposed in (Tao \& Panwar, 2004 \& 2006), where the third dimension models the state of back-off slots between successive transmission periods. The fact that the number of idle slots between successive transmissions can be at most the minimum of AC-specific AIFS values is also considered. This model is able to compute the maximum sustainable throughput and service delay distribution for each priority class when the network is under heavy load.

In (Hui \& Devetsikiotis, 2004, 2005 \& 2006), the authors combined several major analytic approaches, including Bianchi's Markov model, Calì's p-persistent CSMA model and Tay's average-value model for DCF analysis, into one average model, to borrow the strengths from these models and compose a unified performance model of EDCA. In particular, they 
use renewal theory to express the throughput, but assume constant packet-transmission probabilities for different stations taking into account the differentiation of both AIFS and CWmin/CWmax. Another assumption is that each packet-transmission probability only depends on a unique collision probability.

In (Lin \& Wong, 2006) the authors extended the work in (Tay \& Chua, 2001) and used the mean value analysis to evaluate saturation throughput in 802.11e networks. The authors argue that the use of multi-dimensional Markov chains and other non-linear equations lead to high computational complexity. Thus, although some of these models provide good accuracy, the high computational complexity make them unsuitable for real-time control functions such as on-line admission control in IEEE 802.11e networks. Their approach is simpler, and yet it maintains good accuracy.

\subsubsection{Analytic model under non-saturation condition.}

In non-saturation models, the saturation assumptions are released basically following two principal methods; (1) modelling the non-saturated condition with Markov analysis, (2) using queuing theory (Kleinrock, 1975) to evaluate certain performance figures through average or Markov analysis.

To perform Markov analysis under non-saturated conditions slot homogeneity is assumed and the model in (Bianchi, 2000) is extended with necessary extra Markov states and transitions. Similar extensions of (Bianchi, 2000) for the non-saturated analysis of 802.11 DCF are proposed in (Duffy et al., 2005) and in (Alizadeh-Shabdiz \& Subramaniam, 2004 \& 2006). These extensions assume a buffer size for the MAC layer of only one packet. However, such an assumption is shown to lead to significant performance prediction errors for EDCA in the case of larger buffers. An extension of the model provided in (AlizadehShabdiz \& S. Subramaniam, 2004) is given in (Cantieni et al., 2005), where station buffers are assumed infinitely large and the MAC queue is assumed empty with a constant probability, regardless of the back-off stage in which the previous transmission took place. In addition to perform throughput analysis, this model is also able to estimate MAC delay.

In (Engelstad \& Osterboused, 2006), a DTMC model is used to perform delay analysis for both DCF and EDCA, considering the same queue utilization probability as in (Cantieni et al., 2005). While most of the analytical models dealing with delay performance of IEEE 802.11 focus on the prediction of only the mean delay due to the MAC functioning, this model provides analytical predictions of the total delay, which also includes the time spent in queue. Therefore this model can be used by higher layer protocols and applications that are interested in the overall performance of the transmission, including queuing delay.

Several models use queuing theory to carry out 802.11(e) performance analysis in nonsaturated conditions. These models need some independent analysis for the calculation of some figures such as collision and transmission probabilities. In (Tickoo \& Sikdar, a \& b 2004), each 802.11 node is modelled as a discrete time $G / G / 1$ queue to derive the service time distribution. However, such a model is based on the assumption that certain quantities in non-saturated conditions can be approximated well using in-saturation analysis. In (Chen et al., 2006) both $\mathrm{G} / \mathrm{M} / 1$ and $\mathrm{G} / \mathrm{G} / 1$ queue models are used on top of the model provided in (Xiao, 2005), which only considers CW differentiation. The use of M/G/1 queuing model together with a simple non-saturated Markov model to calculate necessary quantities is analysed in (Lee et al., 2006). In (Foh \& Zukerman, 2002) a framework to analyze the performance of DCF under statistical traffic based on Markov chains is presented, where the 
number of contending nodes is modelled as an $\mathrm{M} / \mathrm{Ej} / 1 / \mathrm{k}$ queue. Such work is extended in (Tantra et al., 2006) to take into account EDCA service differentiation. This work analyses both the throughput and delay performance of EDCA mechanism under statistical traffic. However, such an analysis is valid only when all nodes have a MAC queue size set to one packet.

\subsection{HCCA}

Currently only a few analytic works on HCCA exist in literature. In (Rashid et al., 2006) the authors introduced a novel analytic queuing framework that allows the performance of HCCA to be analysed when supporting Variable Bit Rate (VBR) traffic applications. Additionally such a framework is useful to support the design of HCCA scheduler and admission control mechanisms.

In (Ghazizadeh et al., 2009), the authors presented a priority queuing model to analyse medium access in the HCCA by making use of an MAP/PH/1 queue with two types of jobs which are suitable to support a wide range of traffic streams.

\section{Admission Control for EDCA}

The EDCA does not implement any mechanism to provide guarantees to real-time traffic. Nevertheless, several works in the literature, e.g., (Moraes et al., 2006), (Mangold et al.,b 2002) and (Gu \& Zhang, b 2003), proved that the protocol can provide a good level of QoS in terms of delay, jitter and bandwidth under low workload conditions. As a result, limiting the number of traffic flows that can gain access to the channel so as to limit the total workload in the network makes it possible to obtain satisfactory performance.

The selection of which traffic can be admitted without compromising the performance of previously admitted traffic is the task of Admission Control.

The IEEE 802.11e standard (IEEE 802.11, 2007) specifies a mechanism to implement an admission control in EDCA scheme. The admission control applies to any AC when the associated Admission Control Mandatory (ACM) field is activated (in the beacon frame). In that case, all STAs that wish to use this AC must send to the QAP an Add Traffic Stream (ADDTS) request containing the TSPEC of the new flow. After receiving an ADDTS request, the Admission Control, on the basis of the received TSPEC and the current system state, decides whether to:

i) admit the new flow with the requested TSPEC,

ii) suggest a different TSPEC that might be admitted by the network, or

iii) reject the flow.

Once a decision is taken, the Admission Control sends an ADDTS response packet to the corresponding STA, notifying of the decision. When the station receives the response, it decides whether the TSPEC suggested by the AP satisfies or not its traffic requirements. If not, the STA is allowed to schedule another ADDTS request after a period of time. If both the QAP and the QSTA accept, the flow becomes active. When the flow finishes, the QSTA must send a Delete Traffic Stream (DELTS) packet to allow the QAP to release the resources used by this flow.

While the standard describes the message exchange to implement admission control, it does not provide any mechanism to determine how to decide which traffic should be admitted to maintain a desired QoS level. However, in the past few years much research has focused on 
admission control in EDCA. Basically, the existing EDCA admission control algorithms can be classified into two categories: measurement-based admission control and model-based admission control. In the measurement-based schemes, admission control decisions are made on the basis of the continuously measured network conditions such as throughput and delay. On the other hand, the model-based schemes use some analytic model to evaluate certain performance metrics to assess the status of the network.

\subsection{Measurement-Based Admission Control}

In the Distributed Admission Control (DAC) presented in (Xiao \& H. Li, a \& b 2004), the QAP announces the transmission budget within the beacon frame. The transmission budget is the additional amount of time available for either a new flow or existing flows to increase their transmission time per AC in the next beacon interval. To calculate such a budget, the QAP counts the amount of time occupied by the transmissions of each AC during each beacon interval. Then, the QAP calculates the transmission budget for each AC by subtracting the occupied time from the transmissions of the relevant AC. Moreover, each station determines an internal transmission limit per AC within each beacon interval on the basis of the successfully used transmission time during the previous beacon period and the transmission budget announced from the QAP. When the transmission budget for an AC is used up, a new QoS flow will not be able to obtain any transmission time, and existing QoS flows will not be able to increase their transmission time.

Based on the DAC scheme, (Xiao et al., 2004) proposed a two-level protection and guarantee scheme. The purpose of the first-level protection is to protect each existing AC_VO and AC_VI flows from new and existing QoS flows, while the purpose of the second-level protection is to protect QoS flows from best-effort traffic.

For the first-level protection, two enhancements, i.e., tried-and-known and early-protection, are introduced in the original DAC scheme. The tried-and-known is an enhancement of DAC mechanism and is only applied to new flows. Each station keeps track of the throughput and delay performances obtained in previous beacon intervals. If the average throughput and/or delay do not meet the flow requirements, the flow will be rejected. The earlyprotection mechanism prevents two or more new flows from being admitted during the same beacon interval when the budget is below a certain threshold. While this first-level protection is able to protect each existing QoS flow from other QoS flows, it cannot protect QoS flows from best-effort traffic, as this kind of traffic does not need admission control. However, too much best-effort traffic can degrade the performance of QoS flows since many collisions might occur. Therefore, a second-level protection is introduced that dynamically tunes the EDCA parameters of the QSTAs. The basic idea is to increase the initial contention window size and interframe space for best-effort traffic when the number of active stations is large so the number of collisions decreases and the bandwidth available increases. This is controlled by the QAP, that sends such parameters within the beacon frame.

The Threshold-Based Admission Control, proposed in (Gu \& Zhang, a 2003), works in both ad hoc and infrastructure modes, so each station needs to measure the traffic condition on the wireless link and decide whether to transmit or reject traffic flows of a specific AC on the basis of network-load. The authors proposed two different methods to assess the network condition and to implement the admission control:

1. Using relative occupied bandwidth: $T_{B u s y}$ is defined as the amount of time (within a given time period, e.g., a beacon interval) the wireless medium is busy, while the 
$B_{\text {occu }}$ is the relative occupied bandwidth and it indicates at what percentage the wireless medium is being used. The lower and upper thresholds for $B_{\text {occu }}$ to maintain a desired QoS can be obtained from simulations or/and calculations, and are indicated as $B_{l o}$ and $B_{u p}$, respectively. Defining "active $A C^{\prime \prime}$ as each previously admitted $A C$ and "inactive $A C$ " as each $A C$ that has been refused, the criteria to admit data flows can be summarized as follows:

- $B_{\text {occu }} \leq B_{l o}$ : Admit the inactive AC with the highest priority during the next period of $T$.

- $B_{l o} \leq B_{\text {occu }} \leq B_{\text {up }}$ : No action taken.

- $B_{\text {occu }} \geq B_{\text {up }}$ : Stop the transmission of the lowest active AC during the next period of $T$.

2. Using average collision: In this case, instead of using the relative occupied bandwidth, a new parameter, that is the average collision ratio during a fixed sampling period, is employed for admission control. The average collision ratio is defined as $R_{c}=N_{c} / N_{t}$, where $N_{c}$ is the number of collisions that have occurred (estimated through the number of retransmissions) and $N_{t}$ is the total number of transmissions. Similarly, there are two thresholds: the lower threshold $R_{l o}$ and the upper threshold $R_{u p}$. The criteria for admission control is the same as that in the case of using relative occupied bandwidth, with the difference that the new $R_{c}, R_{l o}$ and $R_{u p}$, parameters are used in place of $\mathrm{B}_{o c c u}, \mathrm{~B}_{l o}$ and $\mathrm{B}_{u p}$, respectively.

Although this threshold-based admission control is very easy to implement, the threshold values are difficult to set.

A different approach is the HARMONICA scheme (Zhang \& Zeadally, 2004), which works only in BSS mode. The AP periodically samples the link-layer quality indicator (LQI) parameters, which include drop rate, end-to-end delay and throughput, to dynamically adjust the channel access parameters CWmin, CWmax and AIFS for each traffic class. A simple and flexible technique to avoid congestion is proposed, which employs two different adaptation algorithms:

1) The relative-adaptation algorithm adjusts the relative differences between the channel access parameters of different classes to obtain QoS guarantee.

2) The base-adaptation algorithm synchronously adapts the channel access parameters of all the classes (increase all or decrease all) to achieve high channel utilization.

Whenever a new real-time application requires admission, HARMONICA will select the traffic class $i$ that best matches its QoS requirement and then execute an admission control process. The decision of admission control is based on the throughput requirement (Reqthroughput) of the flow and the monitored LQI parameters. The basic idea is to check whether it is possible to take the required bandwidth from the best effort class ( $B E_{\text {throughput }}$ ) while guaranteeing a minimal bandwidth $\left(B E_{M i n}\right)$ for the best-effort traffic class. In particular, in order to admit a new QoS flow, three requirements need to be satisfied:

1. The relative adaptation has reached a stable state;

2. $B E_{\text {throughput }}-$ Req $_{\text {throughput }} \geq B E_{\text {Mini }}$;

3. The bandwidth in $B E_{\text {throughput }}$ can be "translated" into Class $i$ without loss.

Results provided in (Zhang \& Zeadally, 2004) show that, by dynamically adjusting channel access parameters, it is possible to simultaneously match the QoS requirements, maximally utilize network resources and guarantee a minimal bandwidth for the best-effort traffic. 


\subsection{Model-Based Admission Control}

A model based approach is proposed in (Pong \& Moors, 2003), where admission control is performed on the basis of the predicted achievable throughput for each flow. The estimation of the achievable throughput is based on the two-dimensional Markov Chain model proposed in (Bianchi 2000), the authors extend such a model so as to estimate the throughput of flows belonging to different ACs, each with different channel access parameters and to incorporate the concept of TOXP into the model. The model takes the following as inputs: EDCA parameters (such as CWmin and the back-off stage), the payload of data packets and some statistics on the channel (such as successful transmission probability, collision probability, idle probability).

The admission control is performed by the AP. Before transmitting its data, a station has to make a request to the admission controller (AP) to obtain the desired bandwidth. If the total traffic (already admitted plus the new flow) exceeds the bandwidth limit, then the request is rejected, otherwise it is accepted. In the latter case ACU searches the best parameters for CWmin and TXOP duration (if used), given the required bandwidth of the new stream.

A Contention Window Based admission control is proposed in (Banchs et al.,2003). The aim of this approach is to adjust the $\mathrm{CW}$ values of the different stations so as to reach the goals of admission control. Consider a set of $n$ IEEE 802.11e nodes which is operating with a contention window set $\left\{\mathrm{CW}_{1}, \ldots, \mathrm{CW}_{\mathrm{n}}\right\}$, that satisfies the throughput requirements $\left\{\mathrm{R}_{1}, \ldots, \mathrm{R}_{\mathrm{n}}\right\}$ for all the stations. Denoting $r_{i}$ as the actual throughput experienced by the station $i, r_{i}$ is greater than $R_{i}$ for each $i=1, \ldots, n$. When a new station $(n+1)$ with a throughput requirement of $R_{n+1}$ wishes to join the network, firstly a new contention window set $\left\{\mathrm{CW}_{1}^{\prime}, \ldots, \mathrm{CW}_{\mathrm{n}}^{\prime}, \mathrm{CW}_{\mathrm{n}+1}^{\prime}\right\}$ is calculated using the analytical model proposed in (Banchs et al.,2003), then the same model is used to compute the throughput. If the resulting throughput meets the requirements, the station $(n+1)$ is accepted and the new contention window set is distributed to all the stations. Otherwise, the station $(n+1)$ is rejected.

\section{Improving real-time traffic support in IEEE 802.11e}

Real-time behaviour is one of the main concerns in industrial networking. This section will discuss the most relevant proposals to enhance real-time traffic support in IEEE 802.11e, in both HCCA and EDCA modes.

\subsection{Scheduler and ACU for HCCA}

In the HCCA reference scheduler, both Service Interval and TXOP are fixed values that are recomputed each time a new Traffic Stream (TS) arrives. Several simulation studies, such as (Casetti et al., 2005) and (Yang et al., 2008), show that the reference scheduler is inefficient, especially when dealing with variable bit rate (VBR) traffic. The reason is that all the TSs, although having different characterizations, are polled with the same period and are granted the same transmission time. As a result, the TXOPs are often overestimated and a significant amount of bandwidth may be wasted.

Several works in literature propose new scheduling algorithms to improve the HCCA performance for either constant bit rate (CBR) or VBR traffic. As most of the traffic in industrial applications is $\mathrm{CBR}$, the former algorithms are more suitable with industrial applications. On the contrary, the latter are more suitable for multimedia applications (Voice 
and Video). The most relevant algorithms for CBR traffic are Real-Time HCCA and Group Sequential Communication (GSC).

The Real-Time HCCA (RTH) algorithm (Cicconetti et al, a 2007) is designed to provide realtime support to traffic flows with a given capacity and period. This technique is composed by some offline and online activities. The most complex activities, i.e., the admission control and timescale computation, are performed offline, while the enforcement procedure is performed online.

In the admission control phase, the QAP executes the admission control algorithm each time a QSTA requests to transmit a new TS. Using the TSPEC parameters contained in such requests, the QAP calculates two other parameters for each new TS: the capacity $C_{i}$ and the period $T_{i} . C_{i}$ is the minimum capacity needed to comply with the TS requirements and is calculated as

$$
C_{i}=\left[\frac{\left(R_{i} \cdot T_{i}\right)}{N_{i}}\right] t_{N_{i}}
$$

where the $R_{i}$ is the mean data rate, $N_{i}$ is the nominal SDU size, $t_{N i}$ is the nominal transmission time. The period $T_{i}$ is set equal to the delay bound $D_{i}$ if $D_{i}$ is smaller than $\left[\mathrm{N}_{\mathrm{i}} / \mathrm{R}_{\mathrm{i}}\right]$, otherwise is set to $\left\lfloor\left(\mathrm{R}_{\mathrm{i}} / \mathrm{N}_{\mathrm{i}}\right) \cdot \mathrm{D}_{\mathrm{i}}\right\rfloor \cdot \mathrm{N}_{\mathrm{i}} / \mathrm{R}_{\mathrm{i}}$. Then, the QAP verifies the schedulability of the traffic using the test introduced in (Baker, 1991) in the multi-programmed environment, treating the non-preemptability of transmissions as a critical section. In this case, the minimum critical section bi for a $T_{S i}$ is $b i=t_{N i}+t_{P i}$, where $t_{P i}$ is the poll time for uplink TSs . When $T S_{i}$ is in a critical section, and is thus scheduled instead of the highest priority $\mathrm{TS}_{\mathrm{j}}, \mathrm{TS}_{\mathrm{i}}$ is said to block $\mathrm{TS}_{\mathrm{j}}$. So, the blocking time for a $\mathrm{TS}_{\mathrm{i}}$ is the maximum critical section duration of TSs with a period longer than $\mathrm{TS}_{\mathrm{i}}$, i.e., $\mathrm{B}_{\mathrm{i}}=\max _{\mathrm{j}>\mathrm{i}}\left\{\mathrm{b}_{\mathrm{j}}\right\}$ considering that the TSs are sorted by increasing period duration, i.e., $i>j \rightarrow T_{i} \geq T_{j}$. The schedulability analysis produces the following sufficient condition to determine the set of $n$ schedulable TSs and which has an $\mathrm{O}(\mathrm{n})$ computational complexity:

$$
\frac{B_{i}}{T_{i}}+\sum_{j \leq 1} \frac{C_{j}+\pi_{j}+t_{P_{j}}}{T_{i}} \leq 1 \quad \forall \mathrm{i}: 1 \leq i \leq n
$$

The admission control procedure produces also a set of parameters describing each TS, composed by $\left[\mathrm{i}, \mathrm{t}_{\mathrm{i}}, \mathrm{TXOP}_{\mathrm{i}}\right]$ tuples, where $\mathrm{i}$ is the index of the next QSTA which can access the medium, $t_{i}$ is the polling time and $\mathrm{TXOP}_{\mathrm{i}}$ is the duration of its transmission. The admitted TSs are scheduled in EDF-order.

The online enforcement activity only consists in reading the next entry in the timetable,

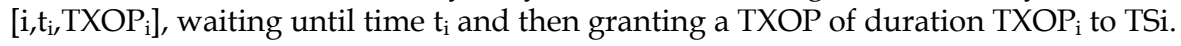

The GSC mechanism presented in (Viègas et al., 2007) is a technique to reduce the polling overhead of the HCCA scheme. The authors propose to grant the channel access to RTstations using a virtual token passing. Stations are classified in two different categories: Real-time and Non Real-time. The GSC procedure groups all the RT stations in a Sequential Group (SG), and identifies them as a vector $\mathrm{L}=\left\{\mathrm{GI}_{1}, \mathrm{GI}_{2}, \ldots, \mathrm{GI}_{\mathrm{np}}\right\}$, where $\mathrm{GI}_{\mathrm{i}}$ is the station identifier and $n p$ is the number of RT stations. Each station maintains a local Sequence 
Counter (SC) that is the image of the distributed variable (SC). A station having the identifier $\mathrm{GI}$ captures the virtual token when $\mathrm{SC}$ is equal to $\mathrm{GI}_{\mathrm{i}}$.

The GSC mechanism works as follows: at the beginning of the CFP, the HC sends a beacon frame and the stations set their NAV, while the SC counter is set to 1. If an RT station is ready to send a frame and the SC is equal to its GI, it is authorized to transmit the real-time message. Once the message has been sent, the other stations listen to the channel and at the first slot-time after the SIFS they increment the SC counter. On the contrary, if a station does not have any frame to send when the SC is equal to its $\mathrm{GI}_{\mathrm{i}}$, then the SC value will be incremented in all the RT stations after a slot-time. This way, the token is implicitly passed to the next RT-stations, until the end of the RT section of L.

The timing analysis provided in (Viègas et al., 2007) shows that the GSC mechanism guarantees a smaller polling overhead than the reference scheduler.

There are several works in literature where new schedulers are investigated to improve the performance of VRB traffic.

In (Grilo et al., 2003), the SETT-EDD scheduling is proposed, that uses the Earliest Due Date policy on the basis of the Estimated Transmission Times and the minimum Service Interval (mSI). The minimum service interval (mSI), i.e., the minimum time that must elapse between two consecutive TXOPs, is specified directly in the TSPEC, while the transmission time is estimated through the maximum burst size given by the TSPEC. Using the arrival time of packets to serve SDUs according to the Earliest Due Date algorithm, the SETT-EDD is shown to perform better than the reference scheduler for the scheduling of VBR traffic.

The Wireless Timed Token Protocol (Cicconetti et al., b 2007) is based on the Timed Token Protocol (TTP) (Grow, 1982), which is a token passing scheme employed as a MAC protocol for ring-based networks.

The token circulation is ruled by the Target Token Revolution Time (TTRT), which is a parameter indicating the reference round duration: its value is computed by the QAP according to the TSPEC values negotiated by the QSTA during the admission control phase. This mechanism provides CBR streams with a fixed capacity, while a minimum reserved rate is allocated for VBR flows.

The Feedback Based Dynamic Scheduler (Boggia et al., 2007) assigns dynamically the TXOP according to queue length estimation, while SI remains fixed. This technique uses the optional piggybacking mechanism to exchange information about the queues together with a discrete time model to estimate the length of uplink TS queues. The duration of the scheduled TXOPs depends on the requirements estimated by the HC during each period.

\subsection{Improving EDCA performance}

Several works in literature proved with simulations (Vittorio et al., a 2007), experimental measurements (Narbutt \& Davis, a \& b 2007) and with analytic models that the EDCA parameters (CWmin, CWmax, AIFS, TXOP) strongly affect the performance of the protocol. Some research works also investigated the effect of EDCA TXOPs on the performance of 802.11e under a saturated scenario. In both (Mangold et al., a 2002) and (Suzuki et al., 2006) the performance analysis is carried out through simulation. In (Tinnirello and S. Choi, 2005) the efficiency of burst transmissions with block acknowledgements is analyzed.

As a result, recent literature presented some mechanisms to dynamically change these parameters on the basis of the channel condition. 
In (Sawaya et al., 2005), the authors proposed an adaptive back-off procedure for EDCA that, instead of using the standard back-off procedure, sets CW[AC] of the back-off timer directly to the most adequate value, calculated on the basis of the congestion level. As in the standard EDCA, the $\mathrm{CW}$ is incremented whenever a station fails to transmit. In fact, the most effective CW values are close to CWmax when the channel congestion is high, while they are close to CWmin when the channel is not congested. The congestion level is assessed through a parameter called ratio and defined as

$$
\text { ratio }=\text { weight } \times \frac{C W_{\text {Current }}-C W_{\min }}{C W_{\max }-C W_{\min }}
$$

where weight is a constant reflecting the accuracy of the channel estimation. The shorter the time between the last transmission and the current estimation, the greater the value of the weight. The new value of the $\mathrm{CW}$ for the next transmission is calculated as

$$
C W_{\text {new }}=\text { ratio } \times\left(C W_{\text {current }}-C W_{\min }\right)+C W_{\min }=\text { weight } \times \frac{\left(C W_{\text {current }}-C W_{\min }\right)^{2}}{C W_{\max }-C W_{\min }}+C W_{\min }
$$

This CWnew value is used as the back-off timer and is shown to be effective in adapting performances to the varying network condition.

In (Romdhani et al., 2004) the authors propose an adaptive mechanism to find the most appropriate values of the back-off parameters for each AC. While the standard EDCA scheme resets the $\mathrm{CW}[\mathrm{AC}]$ value to the $\mathrm{CW}_{\min }[\mathrm{AC}]$ value after each successful transmission, the proposed scheme assesses channel status by taking into account the estimated collision rate, $f_{\text {curr }}^{j}$, whose value is computed as

$$
f_{\text {curr }}^{j}=\frac{E\left(\text { collions }_{j}[p]\right)}{E\left(\text { data }_{-} \text {sent }_{j}[p]\right)}
$$

where $E$ (collisions $s_{j}[p]$ ) is the number of collisions experienced by the station $p$ at step $j$, and $E\left(\right.$ data_sent $\left.t_{j}[p]\right)$ is the total number of packets that have been sent in the same period. To minimize the bias against transient collisions, an Exponential Weighed Moving Average is used to smoothen the estimates values, i.e.,

$$
f_{\text {avg }}^{j}=(1-\alpha) \times f_{\text {curr }}^{j}+\alpha \times f_{\text {avg }}^{j-1}
$$

where $\alpha$ is a constant in $(0,1)$ that determines the memory of the estimation.

To maintain the priority-based discrimination between different classes, a Multifactor Factor $\mathrm{MF}[\mathrm{AC}]$ (on the formula) is defined for each class. This factor is limited to 0.8 and is defined as

$$
M F[i]=\min \left((1+(A C \times 2)) \times f_{\text {avg }}^{j}, 0.8\right)
$$

Using this formula, the highest priority is given to the CW parameter with the smallest MF values. 
After each successful transmission the $\mathrm{CW}$ value is updated as

$$
C W_{\text {new }}[A C]=\max \left(C W_{\min }[A C], C W_{\text {old }}[A C] \times M F[A C]\right)
$$

while after an unsuccessful transmission (collision) of a packet belonging to class $\mathrm{AC}$ the $\mathrm{CW}$ value is updated as

$$
C W_{\text {new }}[A C]=\min \left(C W_{\max }[A C], C W_{\text {old }}[A C] \times P F[A C]\right)
$$

where $P F$ is a parameter proposed in a draft version of the IEEE 802.11e standard to calculate the $\mathrm{CW}_{\text {new }}$ value, and it is used by the authors to ensure that high priority traffic has the smallest $\mathrm{CW}$ values.

In (Vittorio et al., b 2007) a new mechanism is proposed, called a Contention Window Adapter (CWA). Instead of setting the CW to an ideal value within the fixed [CWmin, CWmax] range defined by the standard, which is shown to be inappropriate in many network load conditions, the CWA adjusts the range of the current $\mathrm{CW}$ (i.e. the values of CWmin and CWmax) of all the ACs, on the basis of the workload information, which is estimated on the basis of the retransmission count. Although the CWA is based on empirical rules, it is shown to improve significantly the performance of the Real-Time flows, which are mapped into the AC_VO category. A similar approach is followed in (Vittorio et al., 2008), where a Contention Window Fuzzy Controller (CWFC) uses fuzzy logic in place of the simple empirical rules of the CWA to dynamically find the most appropriate CW range on the basis of the network status, estimated in terms of both global throughput and local retransmissions count.

The Virtual Token Passing-CSMA (VTP-CSMA) architecture has been proposed in (Moraes et al., 2007) to handle the timing constraints of real-time traffic when real-time devices share the wireless channel with devices that are not time constrained. Two different types of stations are considered: RT (Real-Time) and ST (Standard Station). A traffic separation mechanism (TSm) is realized, so that, when a collision occurs, the ST stations select a random back-off interval according to the access category, while the RT stations (that transmit their traffic at the highest priority of EDCA) set both the CWmin and CWmax parameters to zero. This way, if two or more RT stations simultaneously contended for the medium access, they would continuously collide and discard the frame. To avoid this problem, the VTP-CSMA serializes the transmission of the RT stations. At the design phase of the network each RT station is assigned a progressive number, which is used to pass a token between the RT stations. In particular, RT stations continuously listen to the wireless channel and, by counting the number of the elapsed time-slots, each station knows whether the token belongs to it or not. Once a station obtains the token, if it has an RT message to transfer, it can transmit it. When it finishes transmitting, the token will pass to the next station. However, if a station obtains the token while not having any frame to transmit, the token will immediately pass to the next RT station (with the subsequent number).

\section{The IEEE 802.11e standard in industrial environments: case studies}

Several works in literature analyze the suitability of IEEE 802.11e for industrial communications by means of either simulations or real measurements in case-study 
scenarios emulating real industrial applications. In the following some relevant works will be shown, categorized in two classes, i.e., case studies using HCCA and EDCA, respectively.

\subsection{The IEEE 802.11e HCCA standard in industrial scenarios}

In (Karanam et al., 2006) and in (Trsek et al., 2006) the authors evaluated the performance of the HCCA in an protocol industrial automation network with real-time requirements by means of a simulation case study using the network simulator OPNET and compared the results with those obtained with EDCA in terms of latency in various scenarios. The authors assessed the performance of the HCCA protocol in two industrial scenarios. In the former only real-time traffic is present, while in the latter there are both real-time and non real-time traffic. An extended 802.11b (11 Mbps) model of OPNET was used, featuring the reference scheduler described by the IEEE 802.11e standard, hence with TXOP and SI values equal for all the Traffic Streams (TSs).

Two types of TS were considered: downstream and upstream. A PLC is connected directly to an AP through a real-time Ethernet network and generates cyclic data to update the outputs of the remote I/O devices. The I/O nodes sent cyclic data of their inputs to PLC through the WLAN. The traffic flow from node to PLC is defined as upstream and from PLC to node as downstream.

Simulations were performed with a growing number of stations, i.e., from 2 to 80 for EDCA and from 2 to 100 for HCCA. As the maximum number of clients in HCCA is constrained by the chosen service interval (SI), the SI was increased according to the growing number of stations. The size of the packets was set to 40 byte for both TSs.

Results showed that for a small number of stations the delays experienced by HCCA and EDCA are similar, as there is a small number of contentions for the medium. However, when the number of the stations exceeds 25, the EDCA delay increases exponentially due to the large number of collisions and retransmissions. Similar results were obtained maintaining a fixed number of stations while increasing the network load.

The authors conclude that HCCA is more suitable than EDCA for the support of industrial traffic, as EDCA becomes inefficient and unreliable when there is either a large number of stations or high network load, while HCCA remains more predictable and reliable.

\subsection{The IEEE 802.11e EDCA standard in industrial scenarios}

In (Moraes et al., 2006) the authors assess, by means of simulations, an industrial scenario consisting of an open communication environment (OCE), where the traffic from RT stations share the same communication medium with generic multimedia (voice and video) and background traffic from a set of standard (ST) stations. Basically two simulation scenarios are analysed: the small population scenario, which considers the case of 20 stations (10-RT; 10-ST), and the large population scenario, which extends the small population scenario to 50 stations (10-RT; 40-ST). Each station operates at orthogonal frequency division multiplexing (OFDM) PHY mode and the PHY data rate is set to $36 \mathrm{Mbps}$. Each RT station generates 1 packet every $2 \mathrm{~ms}$ with a 45 bytes data payload, while the load offered by ST stations ranges from $5 \%$ to $95 \%$ of the PHY data rate (36 Mbps).

The results showed that the RT stations are able to transfer significantly more packets containing VO traffic than ST stations, even though the same access category is used. Such improvement is due to the TXOP concept introduced in the $802.11 \mathrm{e}$ amendment that defines 
the time interval during which a station is able to transfer a burst of packets from the same access category, after winning the medium access. Consequently, an RT station will be able to transfer a higher number of packets than an ST station using the same access category (VO), because RT-VO packets are shorter than ST-VO packets.

When increasing the number of stations contending for the medium access, there is a degradation of the QoS for large population scenarios.

The authors showed that real-time traffic transferred by RT stations has an average packet delay slightly worse than the voice traffic transferred by ST stations, although RT stations were able to transfer more messages than ST stations.

The authors conclude that the default values of the EDCA parameters are not able to guarantee the timing requirements of industrial communication when the AC_VO class is used to support real-time traffic in shared medium environments and other types of traffic are also present.

In (Cena et al., 2008) the authors performed an in-depth evaluation of the performance achievable by EDCA in industrial environments. The Authors provide a perspective on requirements and characteristics of the traffic typically found in industrial control applications. Four different traffic categories are defined:

- Urgent asynchronous notifications (alarm, RT0);

- $\quad$ Process data sent on a predictable schedule (periodic, RT1);

- $\quad$ Process data sent on a sporadic schedule (RT2);

- Parameterization service (NRT).

RT0 traffic is related to either alarms that are generated spontaneously by devices (failure/error notifications) or asynchronous time-critical commands sent by the application master. RT1 traffic consists of process data characterized by real-time requirements that are generated in a predictable way (periodic traffic). The authors simulate the access to channel of this traffic as a TDMA scheme where the transmission is organized as a repeated communication cycle of fixed duration. Within each cycle, each station sends periodic frame in its assigned slot(s) (e.g. using synchronization). RT2 traffic is similar to RT1 traffic but it is generated in an unpredictable way (aperiodic). Finally, NRT traffic is related to network operations with no particular real-time requirements (e.g., remote configuration, management and diagnostics).

The authors mapped the RT0 on AC_V0 (highest priority), the RT1 on AC_VI, the RT2 on AC_BE and the NRT on AC_BK. The scenario evaluated is composed of 20 stations, 10 of them, defined as "stations under test", that produce a specific kind of traffic and 10, defined as "the interfering stations", that generate low priority traffic. The performance evaluated is the response time, defined as the time elapsed between the transmission request issued at the sender and the receiving time at the intended destination.

The work presents many results obtained by several simulations with different scenario settings. In general, the Authors show that EDCA (enhanced through TDMA techniques to exploit the knowledge about predictable traffic) can be considered a suitable solution for industrial applications, as long as safety and/or time critical requirements are not a primary issue. In fact, the average performance resembles closely those achievable with the currently existing fieldbus networks, but, compared to fieldbuses, WLANs exhibit a noticeably lower degree of determinism. 


\section{Conclusions}

This chapter addressed the case for wireless networks in automation and the significant efforts currently made by a large community of researchers, from both academia and industry, to investigate suitable solutions to adapt the IEEE 802.11e standard to the industrial communication needs on the factory floor.

This chapter provided an overview of current literature concerning the use of IEEE 802.11e in industrial environment, focusing on real-time performance of both EDCA and HCCA mechanisms. The limits of such protocols have been discussed and some notable works to improve their real-time performance have been presented. Such works can be used and combined to improve the support for real-time industrial traffic. As an example, studies on the EDCA admission control algorithms that limit the workload in a wireless network might take advantage of some analytic models predicting the performance of the protocol from the workload and the protocol parameters to provide probabilistic guarantees.

Finally, this chapter discusses the results from case studies that analyse the performance of IEEE 802.11e networks in realistic industrial scenarios.

Despite the significant effort of researchers, there are still some open issues concerning the introduction of wireless local area networks (WLANs) in the factory floor. The most relevant is how to achieve performance guarantees while using an unreliable and non-deterministic wireless channel. Other open issues are: the integration with pre-existing wired networks, so as to form hybrid architectures that are still able to meet the performance requirements; the support for mobility and handover under real-time and reliability constraints; security and privacy of industrial communications; scalability of real-time wireless networks. All these issues are currently object of notable research efforts.

Among these efforts, there is the Flexible Wireless Automation in Real-Time Environments (flexWARE) collaborative project, funded by the European Commission under the 7FP. This project aims at providing real-time communication on the factory floor with wireless local area networks (WLANs), with a special focus on security, flexibility and node mobility.

The outcome of the flexWARE project will be a turnkey system that can overcome the restrictions of the state-of-the-art wireless real-time systems, which are bounded to a single cell, rather than a multiple cell network covering the whole factory, and will define a platform that fulfils the requirements of flexible wireless communications. In the flexWARE architecture, the wireless infrastructure is integrated with a real-time backbone network that can be used to connect different nodes spread over the entire factory floor. Moreover, such an infrastructure can transparently switch between access points. In addition, it can provide time synchronization, location awareness and security. All these features are offered without compromising on the real-time feature of the whole system.

\section{References}

IEEE 802.11b, Part 11: Wireless LAN Medium Access Control (MAC) and Physical Layer (PHY) Specifications: High-speed Physical Layer Extension in the 2.4 GHz Band, Supplement to IEEE 802.11 Standard (Sept. 1999).

IEEE 802.11a, Part 11: Wireless LAN Medium Access Control (MAC) and Physical Layer (PHY) Specifications: High-speed Physical Layer Extension in the $5 \mathrm{GHz}$ Band, Supplement to IEEE 802.11 Standard (Sept. 1999). 
IEEE 802.11g, Part 11: Wireless LAN Medium Access Control (MAC) and Physical Layer (PHY) Specifications: Further Higher-Speed Physical Layer Extension in the 2.4 GHz Band, Supplement to IEEE 802.11 Standard (June 2003).

IEEE Std 802.11TM, IEEE Standards for information Technology, 2007.

Alizadeh-Shabdiz, F. and Subramaniam, S. (2004). "Analytical Models for Single-Hop and Multi-Hop Ad Hoc Networks", Proceedings of the First International Conference on Broadband Networks, pp. 449 - 458, ISBN: 0-7695-2221-1, IEEE Computer Society Washington, DC, USA.

Alizadeh-Shabdiz, F. and Subramaniam, S. (2006). "Analytical Models for Single-Hop and Multi-Hop Ad Hoc Networks," Mobile Networks and Applications, Vol. 11, Issue 1, pp. 75-90, ISSN:1383-469X.

Banchs, A.; Perez-Costa, X. and Qiao, D. (2003). "Providing throughput guarantees in IEEE 802.11e wireless LANs," in Proc. the 18th International Teletraffic Congress(ITC-18), Berlin, Germany.

Baker, T.P.(1991). "Stack-based scheduling of real-time processes". Journal of Real-Time Systems, Vol. 3, No. 1, pp. 67-99, ISSN: 1573-1383, Springer Netherlands

Bianchi, G. (2000). "Performance Analysis of the IEEE 802.11 Distributed Coordination Function", IEEE Journal on Selected Areas in Communications, Volume 18, Issue 3, pp. 535-547.

Boggia, G.; Camarda, P.; Grieco, L. A. and Mascolo, S. (2007). "Feedback-Based Control for Providing Real-Time Services with the 802.11e MAC". IEEE/ACM Transactions on Networking, 15(2):323-333. Volume: 15, Issue: 2 pp. 323-333, ISSN: 1063-6692, San Francisco, CA, USA.

Calì, F.; Conti, M. and Gregori, E. (1998). "IEEE 802.11 Wireless LAN: Capacity Analysis and Protocol Enhancement," in Proc. IEEE Infocom'98. Vol. 1, pp: 142 - 149.

Calì, F.; Conti, M. and Gregori, E. (2000). "Dynamic Tuning of the IEEE 802.11 Protocol to Achieve a Theoretical Throughput Limit," IEEE/ACM Trans. Netw., Vol. 8, Issue 6, pp. 785-799.

Cantieni, G. R.; Ni, Q.; Barakat, C. and Turletti, T. (2005). “Performance Analysis under Finite Load and Improvements for Multirate 802.11," Comp. Commun., Vol. 28, Issue 10, pp. 1095-1109.

Casetti, C.; Chiasserini, C.-F.; Fiore, M. and Garetto, M. (2005). "Notes on the inefficiency of 802.11e HCCA", in IEEE Vehicular Technology Conference VTC 2005. Vol. 4, pp. 25132517.

Cena, G. Bertolotti, I.C. Valenzano, A. Zunino, C. (2008). “Industrial applications of IEEE 802.11e WLANs", IEEE International Workshop on Factory Communication Systems, 2008. WFCS 2008. .pp. 129-138, ISBN: 978-1-4244-2349-1, Dresden.

Chen, X.; Zhai, H.; Tian, X. and Fang, Y. (2006). "Supporting QoS in IEEE 802.11e Wireless LANs", IEEE Trans. Wireless Commun., Vol. 5, Issue 8, pp. 2217 - 2227.

(a) Cicconetti, C.; Lenzini, L.; Mingozzi, E. and Stea, G (2007). "Design and Performance Analysis of the Real-Time HCCA Scheduler for IEEE 802.11e WLANs". Computer Networks, Vol. 51 , Issue 9, pp. 2311-2325, ISSN:1389-1286, Elsevier NorthHolland, Inc. New York, NY, USA. 
(b) Cicconetti, C.; Lenzini, L.; Mingozzi, E. and Stea, G (2007). “An efficient cross layer scheduler for multimedia traffic in wireless local area networks with IEEE 802.11e HCCA". ACM Mob. Comput. and Commun. Vol. 11 , Issue 3, pp. 31 - 46, ISSN:1559-1662, New York, NY, USA.

Duffy, K.; Malone, D. and Leith, D. J. (2005). "Modeling the 802.11 Distributed Coordination Function in Non-Saturated Conditions," IEEE Commun. Lett., Vol. 9, Issue 8, pp.715- 717.

Engelstad, P. E. and Osterbo, O. N. (2006). "Analysis of the Total Delay of IEEE 802.11e EDCA and 802.11 DCF," in IEEE International Conference on Communications, Vol. 2, pp. 552 - 559, ISSN: 8164-9547, ISBN: 1-4244-0355-3, Istanbul.

flexWARE project. Link: http:/ / www.flexware.at/

Foh, C. H. and Zukerman, M. (2002). "A New Technique for Performance Evaluation of Random Access Protocols," in IEEE International Conference on Communications, Vol. 4, pp. 2284 - 2288, ISBN: 0-7803-7400-2.

Ghazizadeh, R.; Fan, P. and Pan, Y. (2009). "A Priority Queuing Model for HCF Controlled Channel Access (HCCA) in Wireless LANs," I. J. Communications, Network and System Sciences, 2009, Vol. 1, pp. 1-89.

Grilo A., Macedo M., and Nunes M, (2003). "A Scheduling Algorithm for QoS Support in IEEE 802.11e Networks", IEEE Wireless Communications, Vol. 10, Issue: 3, pp. 36- 43, ISSN: 1536-1284 .

Grow, R. (1982). "A timed-token protocol for local area networks", In I. Electronic Conventions, editor, Proc. Electro/82, number Paper 17/3 in Token Access Protocols, El Segundo, Calif.

(a) Gu, D. and Zhang, J. (2003). “A new measurement-based admission control method for IEEE 802.11 wireless local area Networks," Mitsubishi Electric Research Laboratory, Tech. Rep. TR-2003-122.

(b) Gu, D. and Zhang, J. (2003). "QoS Enhancement in IEEE 802.11 Wireless Area Networks," IEEE Commun. Mag., vol. 41, no. 6, pp. 120-24. ISSN: 0163-6804.

Hui, J. and Devetsikiotis, M. (2006). "Metamodeling of Wi-Fi Performance," in Proc. IEEE ICC '06, 2006. ICC '06. IEEE International Conference on Communications, Vol. 2, pp. 527-534, ISSN: 8164-9547, ISBN: 1-4244-0355-3, Istanbul.

Hui, J. and Devetsikiotis, M. (2004). "Performance Analysis of IEEE 802.11e EDCA by a Unified Model," in Proc. IEEE Globecom '04, Vol. 2, pp. 754 - 759, ISBN: 0-7803-87945 .

Hui, J. and Devetsikiotis, M. (2005). "A Unified Model for the Performance Analysis of IEEE 802.11e EDCA," IEEE Trans. Commun. Vol. 53, Issue: 9, pp. 1498-1510, ISSN:00906778 .

Inan, I.; Keceli, F. and Ayanoglu, E. (2008), “Analysis of the 802.11e Enhanced Distributed Channel Access Function", CoRR abs/0704.1833.

Karanam, S. P.; Trsek, H. and Jasperneite, J. (2006). "Potential of the HCCA scheme defined in IEEE802.11e for QoS enabled Industrial Wireless Networks," in Proc. 2006 IEEE International Workshop on Factory Communication Systems (WFCS), pp. 227-230, ISBN: 1-4244-0379-0.

Kleinrock L. (1975), “Queueing Systems” . John Wiley and Sons. 
Kong, Z.; Tsang, D. H. K.; Bensaou, B. and Gao, D. (2004). "Performance Analysis of the IEEE 802.11e Contention-Based Channel Access," IEEE J. Select. Areas Commun., Vol. 22, Issue: 10, pp. 2095-2106, ISSN: 0733-8716.

Lee, W.; Wang, C. and Sohraby, K. (2006). “On Use of Traditional M/G/1 Model for IEEE 802.11 DCF in Unsaturated Traffic Conditions," in Proc. IEEE WCNC '06. Vol. 4, pp. 1933-1937, ISSN: 1525-3511, ISBN: 1-4244-0269-7, Las Vegas, NV.

Li, B. and Battiti, R. (2004). "Analysis of the IEEE 802.11 DCF with Service Differentiation Support in Non-Saturation Conditions,", Quality of Service in the Emerging Networking Panorama, Volume 3266, ISBN: 978-3-540-23238-4.

Lin, Y. and Wong, V. W. (2006). "Saturation Throughput of IEEE 802.11e EDCA Based on Mean Value Analysis," in Proc. IEEE WCNC '06, Vol. 1, pp. 475-480, ISSN:15253511, ISBN: 1-4244-0269-7, Las Vegas, NV.

(a) Mangold, S.; Choi, S.; May, P. and Hiertz, G. (2002). “IEEE 802.11e - Fair Resource Sharing Between Overlapping Basic Service Sets," in Proc. IEEE PIMRC '0, Vol. 1, pp. 166- 171, ISBN: 0-7803-7589-0.

(b) Mangold, S.; Choi, S.; May, P. and Hiertz, G. and Stibor, L. (2002). “IEEE 802.11e wireless LAN for quality of service,", Proceedings of the European Wireless, Vol. 1, pp. 32-39, Florence, Italy.

Moraes, R.; Portugal, P. and Vasques, F. (2006). "Simulation Analysis of the IEEE 802.11e EDCA Protocol for an Industrially-Relevant Real-Time Communication Scenario", IEEE ETFA'06, pp. 202-209, ISBN: 0-7803-9758-4, Prague, Czech Republic.

Moraes, R.; Vasques, F.; Portugal, P. and Fonseca, J.A. (2007). “VTP-CSMA: A Virtual Token Passing Approach for Real-Time Communication in IEEE 802.11 Wireless Networks". IEEE Trans. Industrial Informatics, Vol.3 ,Issue: 3, pp. 215-224, ISSN: 1551-3203.

(a) Narbutt, M. and Davis, M. (2007). "Experimental tuning of AIFSN and CWmin parameters to prioritize voice over data transmission in 802.11e WLAN networks". Proceedings of the IWCMC 2007, pp.140-145, ISBN:978-1-59593-695-0, New York, NY, USA.

(b) Narbutt, M. and Davis, M. (2007). “The capability of the EDCA mechanism to support voice traffic in a mixed voice/data transmission over 802.11e WLANs - an experimental investigation,", 32nd IEEE Conference on Local Computer Networks (LNC 2007), pp.463-470. Dublin, Ireland.

Pong, D. and Moors, T. (2003). "Call admission control for IEEE 802.11 contention access mechanism," in Proc. IEEE GLOBECOM'03, vol. 1, pp. 174-178, ISBN: 0-7803-79748, San Francisco.

Rashid, M. M. and Hossain, E. (2006). "Queuing analysis of 802.11e HCCA with variable bit rate traffic," IEEE International Conference on Communications, Vol. 10, pp. 4792 4798, ISSN: 8164-9547, ISBN: 1-4244-0355-3 , Istanbul.

(a) Robinson, J. W. and Randhawa, T. S. (2004). "Saturation Throughput Analysis of IEEE 802.11e Enhanced Distributed Coordination Function," IEEE J. Select. Areas Commun., Vol. 22, Issue: 5, pp. 917-928, ISSN: 0733-8716.

(b) Robinson, J. W. and Randhawa, T. S. (2004). “A Practical Model for Transmission Delay of IEEE 802.11e Enhanced Distributed Channel Access", Proc. IEEE PIMRC'04, Vol. 1, pp. 323-328, ISBN: 0-7803-8523-3. 
Romdhani, L.; Ni, Q. and Turletti, T. (2004). "Adaptive EDCF: Enhanced Service Differentiation for IEEE 802.11 Wireless Ad-Hoc Networks," Wireless Commun. and Mobile Comp. Vol. 2, pp. 1373-1378, ISSN: 1525-3511, ISBN: 0-7803-7700-1, New Orleans, LA, USA.

Sawaya, J.; Ghaddar, B.; Khawam, S.; Safa, H.; Artail, H. and Dawy, Z. (2005) "Adaptive Approach for QoS Support in IEEE 802.11e Wireless LAN," IEEE International Conference on WiMob, Vol. 2, pp. 167-173, ISBN: 0-7803-9181-0.

Steigmann, R. and J. Endresen, R. (2006). Introduction to WISA - Wireless Interface for Sensors and Actuators, White Paper ABB.

Suzuki, T.; Noguchi, A. and Tasaka, S. (2006). "Effect of TXOP-Bursting and Transmission Error on Application-Level and User-Level QoS in Audio-Video Transmission with 802.11e EDCA," in Proc. IEEE PIMRC '06, pp. 1-7, ISBN: 1-4244-0329-4, Helsinki.

Tantra, J. W.; Foh, C. H.; Tinnirello, I. and Bianchi, G. (2006). "Analysis of the IEEE 802.11e EDCA Under Statistical Traffic," in Proc. IEEE ICC '06, Vol. 2, pp. 546-551, ISSN: 8164-9547, ISBN: 1-4244-0355-3. Istanbul.

Tao, Z. and Panwar, S. (2004). "An Analytical Model for the IEEE 802.11e Enhanced Distributed Coordination Function," in Proc. IEEE ICC '04, Vol. 7, pp. 4111- 4117, ISBN: 0-7803-8533-0.

Tao, Z. and Panwar, S. (2006). "Throughput and Delay Analysis for the IEEE 802.11e Enhanced Distributed Channel Access," IEEE Trans. Commun., Vol. 54, Issue: 4, pp. 596- 603, ISSN: 0090-6778.

Tay, J. C. and Chua, K. C. (2001). "A Capacity Analysis for the IEEE 802.11 MAC Protocol," Wireless Netw., Vol. 7, Issue 2, pp. 159 - 171, ISSN:1022-0038, Kluwer Academic Publishers.

(a) Tickoo, O. and Sikdar, B. (2004). "Queueing Analysis and Delay Mitigation in IEEE 802.11 Random Access MAC based Wireless Networks,", Proc. IEEE Infocom '04, Vol. 2, pp. 1404-1413, ISSN: 0743-166X , ISBN: 0-7803-8355-9.

(b) Tickoo, O. and Sikdar, B. (2004). "A Queueing Model for Finite Load IEEE 802.11 Random Access MAC," in Proc. IEEE ICC '04, Vol. 1, pp. 175- 179, ISBN: 0-78038533-0.

Tinnirello, I. and Choi, S. (2005). "Efficiency Analysis of Burst Transmissions with Block ACK in Contention-Based 802.11e WLANs," in Proc. IEEE ICC '05, Vol. 5, pp. 34553460, ISBN: 0-7803-8938-7.

Trsek, H.; Jasperneite, J. and Karanam, S. P. (2006). "A Simulation Case Study of the new IEEE 802.11e HCCA mechanism in Industrial Wireless Networks,", Proc. 11th IEEE International Conference on Emerging Technologies and Factory Automation (ETFA 2006), pp. 921-928, ISBN: 0-7803-9758-4 Prague, Czech Republic

(a) Vittorio, S.; Kaczynski, G. and Lo Bello, L. (2007). "Improving the real time capabilities of IEEE 802.11e through a Contention Window Adapter", RTAS'07_WIP, pp.64-67, Bellevue, USA.

(b) Vittorio, S. and Lo Bello, L. (2007). “An approach to enhance the QoS support to real-time traffic on IEEE 802.11e networks", Proc. of the 6th Intl Workshop on Real Time Networks (RTN 07), Pisa 2007, http://rtn2007.loria.fr. 
Vittorio, S. Toscano, E. Lo Bello, L. (2008). “CWFC: A contention window fuzzy controller for QoS support on IEEE 802.11e EDCA", IEEE International Conference on Emerging Technologies and Factory Automation, 2008. ETFA 2008, pp. 1193-1196, ISBN: 978-1-4244-1505-2, Hamburg.

Viegas Jr., R.; Moraes, R.; Guedes, L. and Vasques, F. (2007) "GSC: A Real-Time Communication Scheme for IEEE 802.11E Industrial Systems". In Proceedings of the 7th IFAC International Conference on Fieldbus Systems and their Applications (FET2007), pp. 111-118, Nov 7-9, Toulouse, France.

Xiao, Y. (2004). "An Analysis for Differentiated Services in IEEE 802.11 and IEEE 802.11e Wireless LANs," Proc. IEEE ICDCS '04, pp. 32 - 39, ISBN:0-7695-2086-3.

Xiao, Y. (2005). "Performance Analysis of Priority Schemes for IEEE 802.11 and IEEE 802.11e Wireless LANs," IEEE Trans. Wireless Commun.,Vol. 4, Issue: 4, pp. 1506- 1515, ISSN: 1536-1276.

(a) Xiao, Y. and Li, H. (2004). "Evaluation of distributed admission control for the IEEE 802.11e EDCA," IEEE Commun. Mag., vol. 42, no. 9, pp. S20-S24, ISSN: 0163-6804.

(b) Xiao, Y. and Li, H. (2004). "Voice and video transmissions with global data parameter control for the IEEE 802.11e Enhance Distributed Channel Access," IEEE Trans. Parallel Distrib. Syst., vol. 15, no. 11, pp. 1041-1053, ISSN:1045-9219.

Yang, G.; Choi, J.; Oh, S. and Lee C. (2008). “An efficient scheduling and admission control algorithm for IEEE 802.11e WLAN", Proceedings of the 2 nd international conference on Ubiquitous information management and communication, pages 12-19, ISBN:978-159593-993-7, Suwon, Korea.

Zhang, L. and Zeadally, S. (2004). "HARMONICA: enhanced QoS support with admission control for IEEE 802.11 contention-based access," in Proc. IEEE RTAS'04, pp. 64-71, ISSN: 1080-1812, ISBN: 0-7695-2148-7, Toronto, Canada. 


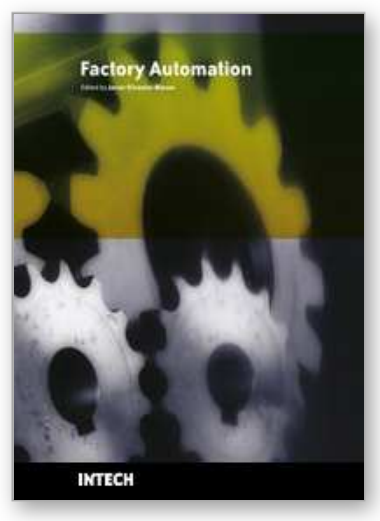

\author{
Factory Automation \\ Edited by Javier Silvestre-Blanes
}

ISBN 978-953-307-024-7

Hard cover, 602 pages

Publisher InTech

Published online 01, March, 2010

Published in print edition March, 2010

Factory automation has evolved significantly in the last few decades, and is today a complex, interdisciplinary, scientific area. In this book a selection of papers on topics related to factory automation is presented, covering a broad spectrum, so that the reader may become familiar with the various fields, and also study them in more depth where required. Within various chapters in this book, special attention is given to distributed applications and their use of networks, since it is one of the most relevant subjects in the evolution of factory automation. Different Medium Access Control and networks are analyzed, while Ethernet and Wireless networks are looked at in more detail, since they are among the hottest topics in recent research. Another important subject is everything concerning the increase in the complexity of factory automation, and the need for flexibility and interoperability. Finally the use of multi-agent systems, advanced control, formal methods, or the application in this field of RFID, are additional examples of the ideas and disciplines that experts around the world have analyzed in their work.

\title{
How to reference
}

In order to correctly reference this scholarly work, feel free to copy and paste the following:

Lucia Lo Bello, Emanuele Toscano and Salvatore Vittorio (2010). A Perspective on the IEEE 802.11e Protocol for the Factory Floor, Factory Automation, Javier Silvestre-Blanes (Ed.), ISBN: 978-953-307-024-7, InTech, Available from: http://www.intechopen.com/books/factory-automation/a-perspective-on-the-ieee-802-11eprotocol-for-the-factory-floor

\section{INTECH}

open science | open minds

\section{InTech Europe}

University Campus STeP Ri

Slavka Krautzeka 83/A

51000 Rijeka, Croatia

Phone: +385 (51) 770447

Fax: +385 (51) 686166

www.intechopen.com

\section{InTech China}

Unit 405, Office Block, Hotel Equatorial Shanghai

No.65, Yan An Road (West), Shanghai, 200040, China

中国上海市延安西路65号上海国际贵都大饭店办公楼 405 单元

Phone: +86-21-62489820

Fax: +86-21-62489821 
(C) 2010 The Author(s). Licensee IntechOpen. This chapter is distributed under the terms of the Creative Commons Attribution-NonCommercialShareAlike-3.0 License, which permits use, distribution and reproduction for non-commercial purposes, provided the original is properly cited and derivative works building on this content are distributed under the same license. 